\title{
A Pilot Study using Imitation and Storytelling Scenarios as Activities for Labelling Emotions by Children with Autism using a Humanoid Robot
}

\author{
Sandra Costa*, Filomena Soares*, Ana Paula Pereira ${ }^{\dagger}$, Cristina Santos $^{*}$ and Antoine Hiolle ${ }^{\ddagger}$ \\ *Email: scosta@dei.uminho.pt \\ R\&D Centre Algoritmi, School of Engineering, University of Minho, Portugal \\ ${ }^{\dagger}$ Institute of Children Studies, University of Minho, Portugal \\ ${ }^{\ddagger}$ Embodied Emotion, Cognition and (Inter-)Action Lab, University of Hertfordshire, United Kingdom
}

\begin{abstract}
In this paper we present a child-robot interaction pilot study, focusing on recognizing and labelling emotions displayed by a humanoid robot. ZECA (Zeno Engaging Children with Autism) has a special skin covering its face which allows the display of facial expressions representing five emotions: joy, sadness, fear, anger, and surprise. These facial expressions were used in two different game scenarios, involving imitation and storytelling activities. The goal of these scenarios is to help the child acquire knowledge about different emotions and to improve their skill in recognizing them. The results show that these scenarios are appropriate for the goal established for this study, and positive behaviours concerning non-verbal communication were observed. This exploratory study demonstrated the possible positive outcomes this child-robot interaction can produce and highlighted the issues regarding data collection and their analysis that will inform future studies.
\end{abstract}

\section{INTRODUCTION}

Autism spectrum disorders (ASD) are characterized according to the criteria in the DSM-5 (Diagnostic and Statistical Manual of Mental Disorders, Fifth Edition) by restrictive patterns of behaviours, repetitive activities or interests and changes in social interaction and communication [1]. About 1 in 68 children has been identified with ASD according to estimates from Autism and Developmental Disabilities Monitoring Network [8]. This neurodevelopmental disorder affects children who then present difficulties when reacting to social stimuli, to imitate behaviours, and to identify their own emotions and those displayed by others [5], [27]. These changes clearly influence the adaptation of the child with ASD to their natural contexts with implications to their cognitive, linguistic, and emotional skills [4]. In the process of building empathy, the identification of emotions displayed by other people is essential [5], and this skill is fundamental for successful social interactions [15].

In this study, we used an expressive humanoid robot with a fully articulated body - ZECA (Zeno Engaging Children with Autism). This robot is able to produce suitable movements and gestures during social interaction. ZECA has human-like features but with a cartoon-like appearance, can produce predictable and repeatable behaviours, showing a great potential for generalization. The research presented in this paper focuses on the use of a humanoid robot to develop emotion recognition and labelling skills in children with ASD. The pilot study described here uses imitation and storytelling scenarios to try and reach this goal. The behavioural repertoire of the robot includes expressive gestures and facial expressions, which can resemble the appearance and movements of a human without trying to create an ultra-realistic appearance.

As we will discover in Section III, robots have already been used with children with ASD to develop their social and communicative abilities with promising results. In this study, the robot mediates the interaction between the child and the experimenter but it is also a tool for teaching. Our main research interest is to assess whether the robot can facilitate the ability to acquire knowledge and skills in recognition and labelling of emotions. The experiments consisted of three sessions with three children diagnosed with ASD. Both qualitative and quantitative measures were used to evaluate the triadic interaction between the children, the robot, and the experimenter.

In this article, we present the analysis of the responses given by the children and the time they took to answer the robot's prompt. Additionally, an analysis of non-verbal communicative behaviours such as pointing, following, imitation, smiling and leaning forwards is presented. This pilot study's main aim is to test the constraints of the two game scenarios with children with ASD. This study is necessary due to the specificity of the target group, and helps us prepare for a larger study which will include a sample of 45 children.

Section II presents some important background elements on how children with ASD perceive facial expressions and emotions. In Section III, we introduce the research projects which also focus on human-robot interactions regarding scenarios about labelling of emotions. Section IV features the procedures used during the experiments. Section V and VI contain the results and the corresponding discussion, respectively. Conclusions and future work are presented in Section VII.

\section{FACIAL EXPRESSIONS RECOGNITION IN ASD}

Empathy is assumed to be composed by three components: motor, emotional and cognitive empathy. Motor empathy 
refers to involuntarily mirroring of other's facial expressions, suggesting the stimulation of shared representations of perception and emotional sharing. Emotional empathy concerns the experience of emotions consistent with and in response to those of others. Cognitive empathy is the capacity to logically comprehend the emotional state of others [2].

Regarding motor emotional responses, some studies have focused on motor mimicry and imitation. A detailed review by Rogers and Pennington [23] underlines a deficit in imitative skills and behaviours in individuals with ASD. Williams et al. linked ASD and an impairment in imitation skills to a malfunction of the mirror neuron system [26]. Children with ASD show a lack of interpersonal coordination of affect [13], lack of emotional expressiveness when requested to imitate affective facial expressions on instruction [14], and difficulties in emotional understanding of faces [21]. They show difficulties in recognizing both basic emotions (such as happiness, fear, anger, surprise and disgust) and more complex ones, which are dependent on the context and culture [10].

In another study, three groups of ten individuals each, matched for verbal mental age and composed of children with ASD in the first, children with Down syndrome in the second, and typically developing children in the last, were tested on a delayed-matching task and on a sorting-by-preference task. Results presented a considerably worse performance from individuals with ASD than from both typically developed and Down participants groups on both facial expression of emotion tasks. However, there were no significant differences between groups on the identity and emotional situation tasks.

Another crucial aspect is the examination of the roles of the verbal and non-verbal sources of information in the ability of participants to recognize emotions. The participants in the study were children with low- and high-functioning ASD, and two other groups with typical developing children, matched by verbal and non-verbal mental age. All participants were shown video clips from which they had to identify the emotions expressed, verbally, non-verbally, or both. The presented emotions were either happy, angry, sad, surprised, or neutral, and verbal expressions of emotion were either explicit, implicit, or neutral, whereas non-verbal expressions were animate or flat. Results showed mostly group differences between higher and lower functioning groups. The performance of low-functioning participants implied they had problems understanding how a person in the video clips felt based on what the person said, if the emotion was not clearly stated. The performance of high-functioning participants suggested that they used more non-verbal than verbal information to determine a speaker's emotion, except when the emotion was explicitly named [16].

The studies presented in this section summarize the research performed with individuals with ASD regarding facial emotion recognition, and they emphasize the common difficulty of this population to identify emotions based on facial expressions. The next section presents robotic-based alternatives which aim to tackle this topic.

\section{Robots AS TOOLS FOR EMOTION RECOGNITION BY CHILDREN WITH ASD}

Only a limited number of projects focuses specifically on the use of robots with children with ASD as promoters of the recognition of facial expressions and emotions.

WikiTherapist project [9] intended to promote incorporation of interaction, through interfaces able to infer intention. The movements were simulated by robots as part of games designed to improve social interaction skills of children with ASD. The humanoid robot FACE [17] was built to allow children with ASD to deal with expressive and emotional information. Together with a shirt with sensors, video cameras and an eye tracking hat, the researchers expected to assess whether children with ASD could learn empathy and especially to better deal with emotional and expressive information. Kismet [3] was designed with the possibility to process a variety of social cues from visual and auditory channels, and exhibited social behaviours to humans with whom it interacted. In their research, the authors studied how children reacted to the robot when it generated a variety of social gestures and facial expressions in response to stimuli from the child. Probo [25] is an animal-like robot, designed to act as a social interface. The authors used Probo, a robot capable of performing basic facial expressions, as a platform to study human-robot interaction. In their opinion, a better recognition of the robot's facial expressions contributes to the general social acceptance. Moreover, the recognition of facial expressions is an important factor for an effective non-verbal communication between a human and a robot.

All these projects offered an important input to the research presented in this paper. However, we believe that the facial characteristics of ZECA will be a key factor for a better generalization of this skill by children with ASD when interacting with other human beings. We aim to address this topic using game scenarios using imitation and storytelling.

Learning by imitation is fundamental to the development of cognitive and social communication behaviours, such as language, play, and joint attention [22]. Imitation is a tool that serves two goals: learning and social function. Storytelling has been used to communicate and transmit general concepts in all sciences, humanities, and to frame an interaction during psychological manipulation. Storytelling is a form of oral communication and serves as a mean of acculturation and transmission of human history [24]. The listener's emotional reactions while paying attention to a story can be, for instance the identification with the story's characters or empathic feeling towards them [20].

\section{Procedure}

Details regarding the robot used in the experiments and the constraints of the game scenarios are presented in the following subsections. In addition, information about the sample that participated in the study, the setup, and the analysis tools are presented. 


\section{A. The Robot}

ZECA is a humanoid robot with the particularity of having a face covered with a polymeric material called Frubber, which allows the display of various facial expressions (Fig. 1). Besides this expressive face, this humanoid robot, developed by Hanson Robotics, possesses a walking body (with 31 degrees of freedom in total), a loud speaker on the chest, and several sensors including two HD cameras in the eyes [12].

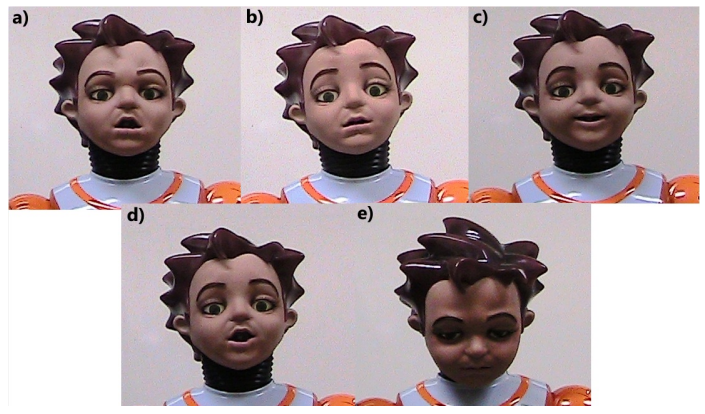

Figure 1. Facial expressions displayed by ZECA. a) anger, b) fear, c) joy, d) surprise, e) sadness

\section{B. Game Scenarios}

The game scenarios evaluated in this study concerned two topics: imitation and storytelling, both of them including an emotional context to tackle the goals previously defined.

Two different scenarios were tested in this study:

- Imitate Me: ZECA performs a facial expression representing one of the following emotions: fear, joy, sadness, surprise, or anger. The child should display on his/her face the same facial expression.

- Storytelling: ZECA tells a social story, where he is the main character. All the stories end with an sentence that characterizes how ZECA feels at the end of the story. The child should show one racket with an image that identifies how ZECA feels.

The facial expressions used in the Imitate Me game scenario (Fig. 1) were previously evaluated by a group of 61 typically developed adults (with the following age range: Mean $(\mathrm{M})=$ 32.393; Standard Deviation $(\mathrm{SD})=9.730$ ) and a group of 42 typically developed children (with the age range: $\mathrm{M}=9.046$; $\mathrm{SD}=0.688$ ) with good efficiency (between 70 and $93 \%$ of correct labelling) [6].

The experiment started with the robot prompting the child to copy it, by saying a sentence such as "Copy my face" or "Imitate my face". The child answered showing the same facial expression with his/her face. In real time, the experimenter used a wireless keypad to classify the answer as either correct or incorrect, and the robot gave the child a reward based on the type of reinforcement the teacher identified as his/her favourite (either movement, verbal, or both). For example, as verbal reinforcement, the robot would say "Very good!" and for a reinforcement with movement, the robot would move its arms in the air. If the answer was incorrect, the robot shook its head and said, for example "Ups. Pay attention. Let's try another one!".

Social stories were especially created based on the work of [11] to provide the children with a context to the emotions felt by a character, in this case ZECA. According to [11]: "a social story describes a situation, skill, or concept in terms of relevant social cues, perspectives, and common responses in a specifically defined style and format". The evaluation of the social stories created is reported in Section V. All the stories in the Storytelling game scenario were accompanied by a visual cue, like the one in Fig. 2.

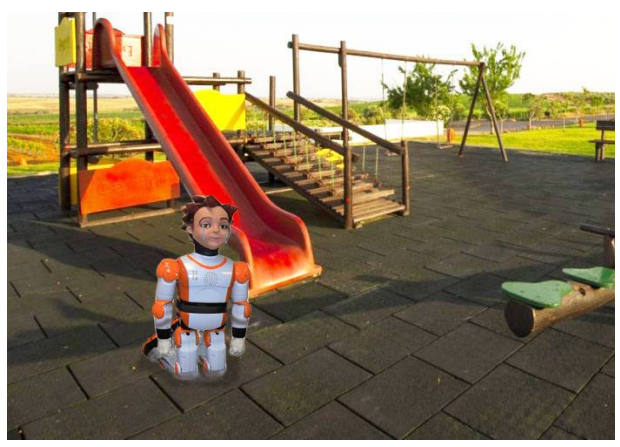

Figure 2. Visual cue shown to the child accompanying the social story.

This individual image for each story was added based on the advice of professionals who interact with children with ASD. As supported in the literature [7], children with ASD appear to mainly use vision as their main input for information. The images were created by a professional designer, and taking into account the plot of each story. An example of the used social stories is: "When I was playing on the playground, I fell on the floor. My arm and my leg were hurting a lot. I had a big scratch, and I could not stop crying". This social story corresponds to the emotion sadness.

For the game scenario Storytelling, the robot started explaining how the game unfolds, told the social story, and prompted the child to choose one of five different rackets (Fig. 3). The visual cue was a picture placed in front of the robot's legs allowing the child to still observe the robot. The rackets, besides the person's face representing an emotion, had a Quick Response (QR) code which identified each emotion. This QR code was then read by one of the HD cameras of the robot. Afterwards, the expression prompted and the answer of the child were matched automatically, and triggered either a correct or incorrect reinforcement on the robot. The procedure for the reinforcement after the child answered to the prompt was the same as described earlier.

To ensure that the images on each racket were representative of an emotion and could be compared with the facial expression displayed by the robot, they were evaluated with an online questionnaire by a group of 67 typically developed adults (with the age range: $\mathrm{M}=28.04 ; \mathrm{SD}=8.22$ ). The analysis of the participants' answers is presented in Section V. The software developed allowed the robot to autonomously identify the answers of the child during the session. This automation 
was considered necessary to help the fluidity of the game and to free the experimenter to interact with the child.

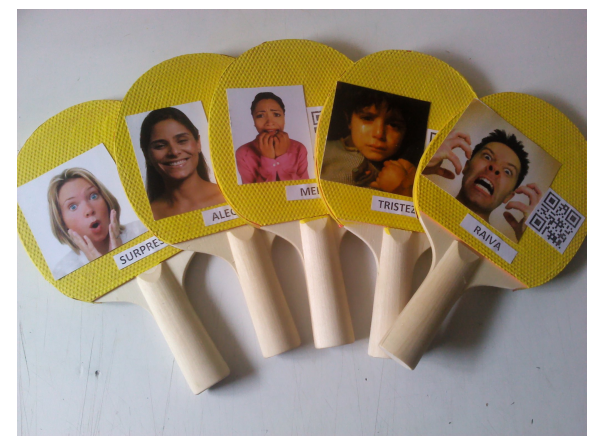

Figure 3. Rackets used in the study for the children to answer the prompts of the robot. Each racket features a picture of a face, a label, and QR code corresponding to an emotion.

During the first and second sessions, both games were played by the children, but only the game Storytelling was played in the third session. The Imitate Me game scenario was not proposed on the last session since all children obtained a perfect score in this activity in the second session.

\section{Participants}

With the goal of applying these scenarios in a wider study (with 45 children), three participants (two girls and one boy with ASD aged thirteen to fifteen years old) participated in this pilot study. The participants are high-functioning, according to their diagnosis (DSM-5), and they all use verbal communication. None of the participants interacted with the robot before, but it is probable they had access to artefacts such as computers or animated toys during their interventions with speech or occupational therapists. The role of the experimenter in the room was to guide the child if necessary, and intervene in case of difficulties. The experiments were carried out by the first author. She introduced the robot to the child, giving them the opportunity to touch it if they wanted. The experimenter ensured that the children did not become agitated or damage the robot during the activity. A signed informed consent form was obtained from all children's parents before they participated in the experiments.

\section{The Setup and Analysis Tools}

The sessions took place in an individual context, encouraging triadic relationships between a child, the experimenter, and the robot. All three sessions were videotaped, with two cameras placed in strategic places to record the interaction of the child with the robot and the experimenter. The produced videos were analysed using the specialized software Observer from Noldus [19] to quantify predetermined behaviours performed by the children during the sessions. The coded behaviours to be evaluated during the interaction were the following: non-verbal communication and the performance in both tasks. Regarding non-verbal communication related behaviours, the literature was consulted to choose the expected behaviours to be observed in children with ASD [18]. The following list presents the chosen behaviours in this category:

- Following: The child follows with a movement of the head (eye gaze if possible) a pointing gesture (with index finger or hand) of the experimenter (even if the pointing gesture is not being performed any more);

- Pointing: The child points at something with the index finger to attract the attention of the experimenter;

- Robot's Imitation: Coded when the child copies movements performed by the robot;

- Experimenter's Imitation: Coded when the child copies movements performed by the experimenter;

- Smiling: Upward curving of the corners of the child's mouth;

- Clapping Hands: The child joining hands together producing a sound;

- Jumping on the chair: The child jumping on his/her chair showing excitement;

- Leaning forwards: The child leans forward towards the robot;

It should be stressed that for following, the emphasis is on behaviours produced after the experimenter directed attention to an object or event, thus establishing a common focus of attention between the child and adult. For pointing, the emphasis is on gestures used by the child to request the experimenter's attention for any object or event. For imitation, repetition was not coded if the child was performing that particular action previously. Verbal repetitions were not coded as imitation. In the activity Imitate Me, copying the face of the robot was not considered imitation, but copying the movement of the robot's body was. For smiling, only the occurrences of clearly expressed smiles were coded.

\section{RESUlts}

Table I shows the results of a study evaluating the emotions conveyed by the stories with 186 typically developed participants recruited on-line. The participants were divided into two groups: younger than 18 years old $(\mathrm{N}=77)$, and older than or aged 18 years old $(\mathrm{N}=109)$. This division was explored having in mind our target population is under aged, but we also wanted to verify if the evaluation performance held across the age groups. On average the participants managed to correctly classify the emotion conveyed by each story with an accuracy of $88.7 \%$, and there are no significant differences between the two groups.

Table I

ANALYSIS OF SOCIAL STORIES BY A TOTAL OF 186 PARTICIPANTS - MEAN (SD).

\begin{tabular}{|c||c||c||c||c|}
\hline & Males & Females & Ages & Rate \\
\hline$<18$ & 9 & 68 & $15.47(2.58)$ & $88.74 \%(19.44)$ \\
$>=18$ & 37 & 72 & $25.06(7.73)$ & $88.75 \%(16.52)$ \\
\hline
\end{tabular}

The ratings achieved by the 67 adults regarding the emotions displayed on each racket show a good efficiency (between 95 
and $100 \%$ of correct labelling). Specifically, the recognition rates were: fear $-95.5 \%$, joy - $100 \%$, sadness - $100 \%$, surprise - $100 \%$, and anger $-98.5 \%$.

Table II presents the mean and the corresponding standard deviation (SD) of the answers given by the children during the three sessions. The answers were classified into "correct", "incorrect" or "no answer". The latter was defined when the robot repeated the prompt by decision of the experimenter. In all the sessions, the children responded correctly more often to the prompts given by the robot, than incorrectly or with no answer. During the Imitate Me game scenario, the right answers varied between $79 \%$ and $100 \%$, and during the Storytelling game scenario between $61 \%$ and $75 \%$.

Table II

PERCENTAGE MEAN OF THE CHILDREN'S ANSWERS IN BOTH GAME SCENARIOS DURING THE THREE SESSIONS (SD)

\begin{tabular}{|l||c||c||c|}
\hline & Correct Answers & Incorrect Ans. & No Ans. \\
\hline Imitate Me 1 & $79.26(20.04)$ & $7.04(6.12)$ & $13.70(15.17)$ \\
Storytelling 1 & $75.40(29.39)$ & $9.52(16.50)$ & $15.08(14.35)$ \\
Imitate Me 2 & $100(0)$ & $100(0)$ & $100(0)$ \\
Storytelling 2 & $61.67(37.53)$ & $19.17(18.76)$ & $19.17(18.76)$ \\
Storytelling 3 & $70.63(37.37)$ & $19.05(32.99)$ & $10.32(9.01)$ \\
\hline
\end{tabular}

Regarding the time the children took to answer the prompt from the robot, the average response time varied between 3.48 and 3.93 seconds $(\mathrm{SD}=0.86)$ in the Imitate Me game and between 6.39 and 6.89 seconds (SD between 2.04 and 2.69) in the Storytelling game scenario.

The videos from the sessions with the children were used to identify behaviours coded as non-verbal communication. Tables III and IV show the results of the frequency and the time used to perform these behaviours. Table III presents the frequency of the behaviours following and pointing, presented previously in the Section IV-D. Only one child did not point during any of the sessions. On average, the behaviour following was observed more often than the behaviour pointing.

Table III

Average Behaviour Occurrence Frequency Per SESSION For the NON-VERBAL COMMUNICATION CATEGORY (SD)

\begin{tabular}{|c||c||c||c|}
\hline & Session 1 & Session 2 & Session 3 \\
\hline Following & $3.67(4.62)$ & $5.33(9.24)$ & $3.33(3.21)$ \\
Pointing & $3.0(5.20)$ & $1(1)$ & $0.67(1.15)$ \\
\hline
\end{tabular}

In table IV, the percentages of time the children imitated either the robot or the experimenter, smile or lean forwards are presented. During all three sessions, all children exhibited the coded behaviours. Moreover, the smiling behaviour was observed more frequently than others, and on average, its frequency and duration increased along the sessions. The behaviour leaning forwards was observed more often in each child's first session. Regarding imitation, the robot was imitated more in the first session, and the experimenter in the last session. In the second session, the time the children spend imitating the robot or the experimenter were similar.
Table IV

MEAN OF THE PERCENTAGE OF TIME THE CHILDREN PERFORMED BEHAVIOURS CODED IN THE NON-VERBAL COMMUNICATION CATEGORY (SD)

\begin{tabular}{|c||c||c||c|}
\hline & Session 1 & Session 2 & Session 3 \\
\hline Robot's Imitation & $0.17(0.3)$ & $0.22(0.38)$ & $0.0(0.0)$ \\
Exp.'s Imitation & $0.0(0.0)$ & $0.25(0.44)$ & $0.28(0.48)$ \\
Smiling & $6.11(5.33)$ & $13.64(13.83)$ & $23.57(18.00)$ \\
Leaning Forwards & $5.68(5.11)$ & $0.12(0.21)$ & $0.16(0.29)$ \\
\hline
\end{tabular}

\section{DISCUSSION}

The results obtained with this pilot study follow the trends identified in the literature (cf. Section III), regarding the use of a robot to mediate interactions between a child with ASD and another person. The scenarios presented here were built based on the difficulties of individuals who belong to this spectrum, and the results show positive evidence of their appropriateness, since the children managed to answer to the robot's prompts, improved their performance, and displayed non-verbal behaviours indicating positive engagement.

The results presented in the previous section show that the children were able to answer to the robot's prompts and to use all the components of the scenarios, such as the display of the emotion by the robot, the use of the racket, the automatic QR Code identification and the corresponding reinforcement. This supports the idea that these scenarios can be used to test the progress of children learning to label different emotions.

The Imitate Me game scenario was interesting to verify that even with difficulties in imitation, the children belonging to this spectrum were able already in the second session to produce $100 \%$ of correct answers. Regarding the game scenario Storytelling, even with a minor efficacy, the children were able to extract the emotion conveyed by the story told by the main character. Regarding the difference in response time between the two scenarios, it may originate from the extra time children needed to process the context of the story, but they still obtained a good efficiency.

While exploring and getting to know the new object and game partner, the children followed the index finger of the experimenter when she tried to attract their attention to the robot. This is a good indication of shared attention between the child and the game partner, and we expect that in the experimental study with more sessions, the child would naturally also point to some detail to attract the experimenter's attention. The analysis of the imitating behaviours displayed by the children during the sessions suggests that the children increasingly transferred the behaviours from the robot to the experimenter, but more sessions are needed to verify this point. The curiosity about the new object might drive them to get closer to the robot, leaning forwards to observe the details of the robot's face, especially its eyes. On average, the time the children smiled during the sessions increased suggesting they were enjoying the task, and further analysis of the children's eye gaze will be interesting to verify at whom the child was looking when exhibiting this behaviour. 


\section{CONCLUSIONS AND FUTURE WORK}

This paper presents the evaluation of two game scenarios which will be included in a larger experimental study. This pilot study presents encouraging results indicating that the use of this robot as a tool to interact with children with ASD can be beneficial for these children. The goal of this study was to verify if the two game scenarios would help the children acquire knowledge about labelling emotions, while at the same time promote child-robot interaction and increasingly childadult interaction. However, due to the small size of the sample used in this study, the entire spectrum of the disorder might not be entirely represented. Additionally, the experimenter had to adjust to the individual differences between the children, mainly constituted by their differences in attention span, which might have resulted in slight variations in the experimental procedure during the sessions.

The results show that the children showed a good performance regarding their answers to the robot's prompts in both scenarios, and the response time decreased along the sessions. Regarding the non-verbal communication, the robot was a useful tool to promote positive behaviours such as smiling and leaning forwards. The robot was also used as an object of imitation which was shortly transferred to the experimenter.

The future work involves a experimental study with a sample of 45 children. These children will be divided into three groups, performing the game scenarios with the robot, without the robot, and a control group. The research presented in this paper is included in the work plan of this experimental study with the aim of evaluating if and how a humanoid robot can be used to teach children with ASD to recognize and label emotions.

\section{ACKNOWLEDGEMENT}

This work has been supported by FCT Fundacao para a Ciencia e Tecnologia in the scope of the project: PEst-OE/EEI/UI0319/2014. The authors are grateful to the Portuguese Foundation (FCT) for the R\&D project RIPD/ADA/109407/2009 and SFRH/BD/71600/2010 scholarship.

\section{REFERENCES}

[1] American Psychiatric Association, Diagnostic and statistical manual of mental disorders: DSM-5, 5th ed. Washington, DC: Autor, 2013.

[2] R. J. R. Blair, "Responding to the emotions of others: Dissociating forms of empathy through the study of typical and psychiatric populations," Consciousness and cognition, vol. 14, no. 4, pp. 698-718, 2005.

[3] C. L. Breazeal, "Sociable machines: expressive social exchange between humans and robots," Ph.D. dissertation, Massachusetts Institute of Technology, 2000.

[4] T. Charman and W. L. Stone, Social and communication development in autism spectrum disorders: Early identification, diagnosis, and intervention. Guilford Press, 2006.

[5] T. F. Clark, P. Winkielman, and D. N. McIntosh, "Autism and the extraction of emotion from briefly presented facial expressions: Stumbling at the first step of empathy." Emotion, vol. 8, no. 6, p. 803, 2008.

[6] S. Costa, F. Soares, and C. Santos, "Facial expressions and gestures to convey emotions with a humanoid robot," in Social Robotics, G. Herrmann, M. Pearson, A. Lenz, P. Bremner, A. Spiers, and U. Leonards, Eds. Springer, 2013, pp. 542-551.
[7] S. Dettmer, R. L. Simpson, B. S. Myles, and J. B. Ganz, "The use of visual supports to facilitate transitions of students with autism," Focus on Autism and Other Developmental Disabilities, vol. 15, no. 3, pp. 163-169, 2000.

[8] Developmental, Disabilities Monitoring Network Surveillance Year 2010 Principal Investigators, "Prevalence of autism spectrum disorder among children aged 8 years-autism and developmental disabilities monitoring network, 11 sites, United States, 2010." Morbidity and mortality weekly report. Surveillance summaries (Washington, DC: 2002), vol. 63, p. 1, 2014.

[9] J. Gillesen, S. Boere, and E. Barakova, "Wikitherapist," in Proceedings of the 28th Annual European Conference on Cognitive Ergonomics. ACM, 2010, pp. 373-374.

[10] O. Golan, S. Baron-Cohen, and Y. Golan, "The reading the mind in films task [child version]: Complex emotion and mental state recognition in children with and without autism spectrum conditions," Journal of autism and developmental disorders, vol. 38, no. 8, pp. 1534-1541, 2008.

[11] C. A. Gray and J. D. Garand, "Social stories: Improving responses of students with autism with accurate social information." Focus on Autistic Behavior, 1993.

[12] D. Hanson, S. Baurmann, T. Riccio, R. Margolin, T. Dockins, M. Tavares, and K. Carpenter, "Zeno: a cognitive character," in AI Magazine, and special Proc. of AAAI National Conference, Chicago, 2009.

[13] C. Kasari, M. Sigman, P. Mundy, and N. Yirmiya, "Affective sharing in the context of joint attention interactions of normal, autistic, and mentally retarded children," Journal of autism and developmental disorders, vol. 20, no. 1, pp. 87-100, 1990.

[14] T. Langdell, "Recognition of faces: An approach to the study of autism," Journal of child psychology and psychiatry, vol. 19, no. 3, pp. 255-268, 1978.

[15] J. M. Leppanen and C. A. Nelson, "The development and neural bases of facial emotion recognition," Advances in child development and behavior, vol. 34, pp. 207-246, 2006.

[16] K. A. Loveland, B. Tunali-Kotoski, Y. R. Chen, J. Ortegon, D. A. Pearson, K. A. Brelsford, and M. C. Gibbs, "Emotion recognition in autism: Verbal and nonverbal information," Development and Psychopathology, vol. 9, no. 03, pp. 579-593, 1997.

[17] D. Mazzei, L. Billeci, A. Armato, N. Lazzeri, A. Cisternino, G. Pioggia, R. Igliozzi, F. Muratori, A. Ahluwalia, and D. De Rossi, "The face of autism," in RO-MAN, 2010 IEEE. IEEE, 2010, pp. 791-796.

[18] P. Mundy, M. Sigman, J. Ungerer, and T. Sherman, "Defining the social deficits of autism: the contribution of non-verbal communication measures," Journal of child psychology and psychiatry, vol. 27, no. 5, pp. 657-669, 1986.

[19] L. Noldus, "The observer: A software system for collection and analysis of observational data," Behavior Research Methods, Instruments, \& Computers, vol. 23, no. 3, pp. 415-429, 1991.

[20] K. Oatley, "A taxonomy of the emotions of literary response and a theory of identification in fictional narrative," Poetics, vol. 23, no. 1, pp. 53-74, 1995.

[21] S. Ozonoff, B. F. Pennington, and S. J. Rogers, "Executive function deficits in high-functioning autistic individuals: relationship to theory of mind," Journal of child Psychology and Psychiatry, vol. 32, no. 7, pp. 1081-1105, 1991.

[22] J. Piaget, Piagets theory. Springer, 1976.

[23] S. J. Rogers and B. F. Pennington, "A theoretical approach to the deficits in infantile autism," Development and Psychopathology, vol. 3, no. 02, pp. 137-162, 1991.

[24] R. C. Roney, "Back to the basics with storytelling," The Reading Teacher, vol. 42, no. 7, pp. 520-523, 1989.

[25] J. Saldien, K. Goris, B. Vanderborght, J. Vanderfaeillie, and D. Lefeber, "Expressing emotions with the social robot probo," International Journal of Social Robotics, vol. 2, no. 4, pp. 377-389, 2010.

[26] J. H. Williams, A. Whiten, T. Suddendorf, and D. I. Perrett, "Imitation, mirror neurons and autism," Neuroscience \& Biobehavioral Reviews, vol. 25, no. 4, pp. 287-295, 2001.

[27] L. Zwaigenbaum, S. Bryson, T. Rogers, W. Roberts, J. Brian, and P. Szatmari, "Behavioral manifestations of autism in the first year of life," Intl. Journal of Developmental Neuroscience, vol. 23, no. 2, pp. 143-152, 2005. 